\title{
Commentary
}

\section{Rapid diagnostic testing for antimicrobial stewardship: Utility in Asia Pacific}

\author{
Anucha Apisarnthanarak MD ${ }^{1}$ (]) Hong Bin Kim MD, FIDSA², Luke Moore PhD ${ }^{3,4,5}$, Yonghong Xiao MD, $\mathrm{PhD}^{6}$, \\ Sanjeev Singh $\mathrm{MD}^{7}$, Yohei Doi MD, $\mathrm{PhD}^{8,9}$, Andrea Lay-Hoon Kwa PharmD ${ }^{10,11}$, \\ Sasheela Sri La Sri Ponnampalavanar MD², Qing Cao MD ${ }^{13}$, Shin-Woo Kim MD, PhD ${ }^{14}$, Hyukmin Lee MD, PhD ${ }^{15,16}$ and \\ Pitak Santanirand $\mathrm{PhD}^{17}$ \\ ${ }^{1}$ Infectious Diseases Division, Thammasat University Hospital, Pathum Thani, Thailand, ${ }^{2}$ Internal Medicine, Seoul National University Bundang Hospital, Seoul \\ National University College of Medicine, Seongnam, Republic of Korea, ${ }^{3}$ Infectious Diseases \& Clinical Microbiology, Chelsea \& Westminster NHS Foundation \\ Trust, London, United Kingdom, ${ }^{4}$ Medical Microbiology, North West London Pathology Imperial College Healthcare NHS Trust, London, United Kingdom, \\ ${ }^{5}$ Imperial College, London, United Kingdom, ${ }^{6}$ State Key Lab for Diagnosis and Treatment of Infectious Diseases, 1st Affiliated Hospital, School of Medicine, \\ Zhejiang University, Hangzhou, China, ${ }^{7}$ Hospital Administration and Medical Services, Amrita Institute of Medical Sciences, Amrita University, Ponekkara, Kochi, \\ Kerala, India, ${ }^{8} \mathrm{~S} c h o o l$ of Medicine, University of Pittsburgh, Pittsburgh, Pennsylvania, United States, ${ }^{9}$ Departments of Microbiology and Infectious Diseases, Fujita \\ Health University School of Medicine, Toyoake, Japan, ${ }^{10}$ Pharmacy Department, Singapore General Hospital, Singapore, ${ }^{11}$ Emerging Infectious Diseases Program, \\ Duke-National University of Singapore Medical School, Singapore, ${ }^{12}$ Department of Medicine, Faculty of Medicine, University of Malaya, Kuala Lumpur, Malaysia, \\ ${ }^{13}$ Shanghai JiaoTong University Affiliated Shanghai Children's Medical Center, Shanghai, China, ${ }^{14}$ Internal Medicine, Kyungpook National University Hospital, \\ Daegu, Republic of Korea, ${ }^{15}$ Department of Laboratory Medicine, Yonsei University College of Medicine, Seoul, Korea, ${ }^{16}$ Korean Society for Laboratory Medicine, \\ Seoul, Republic of Korea and ${ }^{17}$ Clinical Pathology, Faculty of Medicine, Ramathibodi Hospital, Mahidol University, Bangkok, Thailand
}

\begin{abstract}
Rapid diagnostic testing (RDT) can provide prompt, accurate identification of infectious organisms and be a key component of antimicrobial stewardship (AMS) programs. However, their use is less widespread in Asia Pacific than western countries. Cost can be prohibitive, particularly in less resource-replete settings. A selective approach is required, possibly focusing on the initiation of antimicrobials, for differentiating bacterial versus viral infections and identifying locally relevant tropical diseases. Across Asia Pacific, more data are needed on RDT use within AMS, focusing on the impact on antimicrobial usage, patient morbidity and mortality, and cost effectiveness. Moreover, in the absence of formal guidelines, regional consensus statements to guide clinical practice are warranted. These will provide a regionally relevant definition for RDT; greater consensus on its role in managing infections; advice on implementation and overcoming barriers; and guidance on optimizing human resource capacity. By addressing these issues, the outcomes of AMS programs should improve.
\end{abstract}

(Received 17 March 2021; accepted 25 March 2021; electronically published 15 June 2021)

Rapid diagnostic testing (RDT) methods offer prompt, accurate identification of infectious organisms and assessment of antimicrobial susceptibility. ${ }^{1}$ RDTs could therefore be a valuable component of the coordinated interventions that comprise multimodal antimicrobial stewardship (AMS) programs. ${ }^{2-4}$

Despite the increasing availability and scope of these methods, there is currently no global or regional consensus on what constitutes an RDT. Indeed, even the definition of 'rapid' within this context has not yet been standardized. A 2017 meta-analysis included any appropriate diagnostic tests that could provide results within 24 hours. ${ }^{5}$ However, throughput times have continued to decrease, and a more stringent definition may now be warranted.

Author for correspondence: Anucha Apisarnthanarak, E-mail: anapisarn@yahoo.com Cite this article: Apisarnthanarak A, et al. (2021). Rapid diagnostic testing for antimicrobial stewardship: Utility in Asia Pacific. Infection Control \& Hospital Epidemiology, 42: 864-868, https://doi.org/10.1017/ice.2021.149
Ideally, RDTs should possibly provide results to the clinician within 4-6 hours. However, in settings where this is not possible, delivery of results within 24 hours may be acceptable. A preferred RDT can yield results to guide treatment before the second dose of antimicrobial is administered. Multiple technologies may fit within this definition, including peptide nucleic acid fluorescent in situ hybridization (FISH), matrix-assisted laser desorption/ ionization-time of flight (MALDI-TOF) mass spectrometry, polymerase chain reaction (PCR), nanoparticle probe technology, lateral-flow enzyme-linked immunoassays (ELISA or LFA), nuclear magnetic resonance, and computed tomography. ${ }^{1,4,6}$

The definition of an RDT should be independent of where the test is conducted, which might be near the patient (possibly at the bedside) or further away (an offsite laboratory), depending on the specific technology used. Also, although multiple technical platforms could potentially be considered RDTs, any definition should incorporate the identification not just of bacterial organisms but also nonbacterial pathogens, to help reduce unnecessary antimicrobial

(c) The Author(s), 2021. Published by Cambridge University Press on behalf of The Society for Healthcare Epidemiology of America. This is an Open Access article, distributed under the terms of the Creative Commons Attribution-NonCommercial-NoDerivatives licence (https://creativecommons.org/licenses/by-nc-nd/4.0/), which permits non-commercial re-use, distribution, and reproduction in any medium, provided the original work is unaltered and is properly cited. The written permission of Cambridge University Press must be obtained for commercial re-use or in order to create a derivative work. 


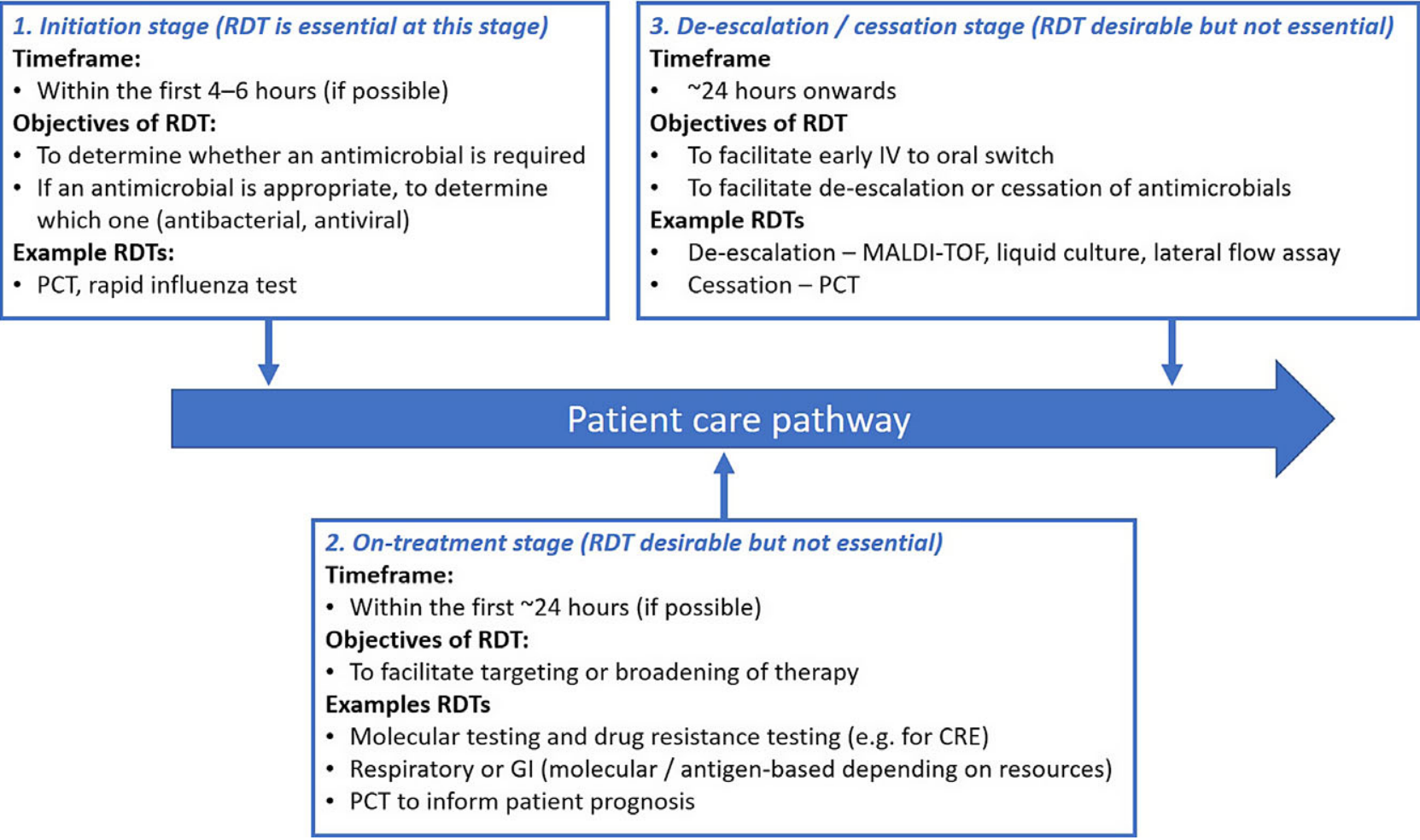

Fig. 1. Impact of RDT on AMS at different time points during the patient journey. Note. AMS, antimicrobial stewardship; CRE, carbapenem-resistant Enterobacteriaceae; GI, gastrointestinal; IV, intravenous; MALDI-TOF, matrix-assisted laser desorption/ionization-time of flight; PCT, procalcitonin; RDT, rapid diagnostic testing.

usage. Furthermore, RDT implementation must be tailored to the specific setting, particularly in the Asia Pacific region, which encompasses countries with wide-ranging economic development levels and many different infective pathogens. Other relevant factors include high specificity and the cost-effectiveness of the technology.

Within an AMS framework, the key advantage of RDT is the facilitation of rational use of antimicrobials in general, and of antibacterials in particular. In considering the patient pathway, RDTs may potentially impact 3 key antimicrobial decision nodes: at initiation, on treatment, and for de-escalation or cessation of treatment (Fig. 1). RDTs are particularly essential at initiation, whereas at other stages they might be considered as desirable rather than essential, because 'nonrapid' diagnostic tests are more affordable (particularly in less resource-replete settings).

In addition to facilitating AMS, RDT can improve individual patient outcomes. A recent meta-analysis by Timbrook et $\mathrm{al}^{5}$ identified 31 studies that compared conventional microbiological methods with molecular RDT in patients with bloodstream infections (BSIs). Their analysis revealed that mortality risk was significantly reduced with RDT versus conventional laboratory techniques (odds ratio [OR], 0.66; 95\% confidence interval [CI], 0.54-0.80). RDT was also associated with reductions in time to effective therapy and length of hospital stay (LOS). ${ }^{5}$

Further stratification showed that significant mortality benefit was evident when RDTs were coupled with an AMS program (OR, 0.64; 95\% CI, 0.51-0.79) but was lost in the absence of AMS (OR, 0.72; 95\% CI, 0.46-1.12). This finding suggests that appropriate team members within AMS programs play a key role in interpreting and acting on RDT data. Ideally, these teams should include members from multiple specialties (eg, infectious disease physicians, clinical pharmacy specialists, microbiologists, nurses, infection control professionals, information system specialists, and hospital epidemiologists). ${ }^{1}$ However, bringing together such broad expertise may be a significant challenge in low- and middle-income countries (LMICs) in Asia Pacific. A recent Korean analysis estimated that the human resource requirement for AMS activities for hospitalized patients requiring antibiotic treatment was $\sim 1.2$ full-time equivalents per 100 beds, ${ }^{7}$ a substantial commitment. Notably, most studies included in the aforementioned Timbrook meta-analysis were observational and quasi-experimental, and more data are needed from randomized controlled trials comparing RDT with conventional laboratory methods. ${ }^{1,5}$ Furthermore, fewer studies have analyzed the use of RDT in patients with non-BSI infections, ${ }^{1}$ and high-quality trials are required in these other disease settings (eg, respiratory tract, gastrointestinal, and central nervous system [CNS] infections).

Many novel RDT methods are associated with elevated capital and consumable costs, and financial outlays may also be required for hiring and training personnel to operate the equipment and interpret the results. There might also be additional challenges around service logistics (eg, all hours vs working hours only; in-batch vs on-demand testing), and these might have a substantial impact on the effectiveness of an AMS program.

The availability of resources is therefore essential, and costeffectiveness analyses will be crucial to determining the value of RDTs in clinical practice. Such analyses may be particularly pertinent in LMICs, including those in the Asia Pacific region, where budgets are often constrained. In the BSI meta-analysis, financial measures were not assessed, although the decrease in LOS suggested potential for reduced cost. ${ }^{5}$ Relatively few cost-effectiveness analyses of RDT have been performed, and most did not adopt a wholehealthcare-economy perspective. A US study of MALDI-TOF for 
rapid identification of BSI organisms, alongside a dedicated AMS program, demonstrated a decrease in total hospital costs of $>$ US $\$ 2,000$ per infection. ${ }^{8}$ This decrease was driven primarily by decreased LOS in the intensive care unit. ${ }^{8}$ In addition, a comprehensive analysis of various RDT platforms demonstrated that cost-effectiveness improvements were particularly significant when combined with AMS, with savings of up to US $\$ 30,000$ per quality-adjusted life year. ${ }^{9}$

Current guidelines for implementing AMS from the Infectious Diseases Society of America encourage the use of RDT in selected patients with BSIs or acute respiratory infections. ${ }^{2}$ More pertinently to much of the Asia Pacific region, a recent AMS tool kit for LMICs developed by the World Health Organization (WHO) highlighted the importance of cost-effective RDTs. Specifically, the WHO document noted that, "There is a great need for affordable, sensitive, specific and rapid diagnostic tests that provide prescribers with quality-assured information about whether or not a patient has a bacterial infection, and which antibiotics the causative bacteria are susceptible to." 3 Nonetheless, there are significant challenges in meeting this aspiration in Asia Pacific.

\section{Current status and challenges in implementing RDT and AMS in Asia Pacific}

The use of RDT as part of AMS programs is not as widespread in Asia Pacific as it is in North America or western Europe. Nonetheless, in high-income countries (HICs) within the region, RDT is increasingly becoming part of standard practice, for example, based on microbiology, antigen testing, biochemistry (particularly procalcitonin [PCT]), and some molecular methods. In a survey of AMS programs in Korean hospitals, almost all had access to some rapid testing (particularly for influenza viruses). ${ }^{10}$ The situation in LMICs in Asia Pacific is more variable, but some RDTs are available in at least some centers.

Realistically, cost is likely to remain prohibitive in many institutions, particularly in LMICs, and a selective approach will be required. Here, resources may be most effectively deployed by focusing on RDTs that assist with the 'initiation' stage of antimicrobial use (Fig. 1), in particular, for differentiating bacterial versus viral infection (eg, PCT, influenza panels, CNS panels, SARS-CoV-2 testing) and for identifying tropical diseases relevant to specific countries or local areas (eg, malaria, dengue, tuberculosis).

Across the Asia Pacific region, data on the use of RDT are lacking. Indeed, of the 31 studies included in the 2017 BSI meta-analysis, ${ }^{5}$ only 2 were from this region: 1 performed in the Republic of Korea ${ }^{11}$ and 1 from Japan. ${ }^{12}$ A small number of studies have followed, particularly in Japan, ${ }^{13,14}$ and analyses from less resource-replete Asian settings have now started to appear in the literature. For example, a recent study demonstrated the utility of a multiplex PCR system for diagnosing CNS infections in a tertiary-care center in India. ${ }^{15}$ In addition, a randomized controlled trial in Vietnam compared rapid pathogen identification using MALDI-TOF versus conventional methods. ${ }^{16}$ However, this study demonstrated no difference in the proportion of patients on optimal antimicrobial therapy within 24 or 48 hours of positive culture. No concomitant AMS program was in place within the trial centers, ${ }^{16}$ suggesting that such initiatives may be as crucial to the effectiveness of RDT in LMICs as they are in HICs.

In addition, to the best of our knowledge, no published data are available on the cost effectiveness of RDT methods in the Asia Pacific region, although studies are ongoing and results are
Table 1. Key challenges to overcome in implementing RDT and AMS in Asia Pacific

Key challenges
- Insufficient funding of, and insufficient access to, some or all RDT
technologies
- Inability of some RDT platforms to accommodate the full range of
relevant organisms, particularly where these differ from North America
and Europe (eg, tropical diseases)
- A lack of microbiology laboratories with sufficient internal expertise
and/or external quality assurance
- Suboptimal patient care pathways and reporting structures that hinder
the process of obtaining rapid test results and subsequent
implementation of findings
- A lack of guideline recommendations and general guidance from
professional societies, which compounds the lack of awareness and
education among physicians regarding RDT and AMS outside of
hospital intensive care and infectious disease departments

Note. AMS, antimicrobial stewardship; RDT, rapid diagnostic testing.

expected soon. Such data are essential if the potential advantages of these techniques are to be realized. Hence, overall, there remains a need for further trials of RDT technologies in Asia Pacific, ideally conducted within the context of AMS programs. Key analyses should include their impact on antimicrobial usage patterns, patient morbidity and mortality, time to effective therapy, LOS and cost effectiveness.

Furthermore, there is a pressing need to improve guidance on developing appropriate AMS programs in Asia Pacific, including direction on the necessary human resources and the requirement for sustainable funding models, applicable both in hospitals and in community settings. Such guidance has already been developed for resource-replete countries of western Europe and North America. ${ }^{17,18}$ However, considerations may be different in Asia Pacific, particularly in LMICs, and should be defined in detail.

Beyond these issues, many other challenges in the Asia Pacific region are likely to be at least somewhat specific to individual countries and territories (Table 1). ${ }^{3,19}$ They include challenges related to technological access and expertise, clinical guidance, and care pathways.

\section{Impact of the SARS-CoV-2 pandemic on RDT and AMS in Asia Pacific}

The SARS-CoV-2 pandemic has had a substantial impact on healthcare provision across the region, drawing resources away from other services including AMS programs. Furthermore, although SARS-CoV-2 is infrequently associated with respiratory bacterial and fungal coinfection, broad-spectrum empirical antimicrobials are often used. ${ }^{20,21}$ Thus, appropriate AMS activities are urgently required.

However, the pandemic has also driven an increased focus on (and uptake of) diagnostic methods that provide rapid and accurate results, particularly PCT, PCR, and molecular testing. More generally, the pandemic has substantially raised overall awareness of infectious diseases among healthcare stakeholders, including clinicians, payers, policy makers, and the public.

As long as 'COVID fatigue' can be overcome, this enhanced awareness creates an opportunity for a renewed discussion among these stakeholders about the broad value of RDT and AMS. 
To maximize the impact of such dialog, there should be an increased focus on generating local data that support the use of RDT, including antibiotic usage patterns, clinical outcomes, and cost-effectiveness analyses.

\section{The need for guidance: Creating consensus on RDT practices in Asia Pacific}

Greater consensus is needed regarding the role of RDT technologies in managing infection in Asia Pacific and regarding the value of RDT in optimizing antimicrobial use within local, national, and regional AMS programs. In the absence of formal guidelines, we are currently developing consensus-based statements to guide clinical practice on the role of RDT in AMS.

These consensus statements will provide a working definition for RDT that is meaningful and appropriate to Asia Pacific settings. We will recommend an inventory of RDTs appropriate for both HICs and LMICs, and current barriers to the use of RDT in these settings will be discussed, together with possible solutions. The consensus statements will provide guidance on implementation of RDTs within current patient pathways (ideally based on point-of-care testing), as well as advice for practice on how to organize and build capacity of faculty and staff for implementing RDT.

The consensus statements will target not only infectious diseases specialists but also other physicians (eg, surgeons, intensive care physicians and general practitioners) and nonphysician healthcare professionals (eg, nurses and pharmacists) routinely managing infections in Asia Pacific to ensure that this guidance reaches a multidisciplinary audience.

Beyond these efforts, it takes a village to devise, implement, and monitor AMS strategies effectively and successfully. Thus, all stakeholders in Asia Pacific need to recognize the utility and potential benefits of RDT in AMS and take action to incorporate RDT to assist AMS efforts wherever it may be beneficial. Greater data collection on the use of RDT that focuses on antimicrobial usage patterns, patient mortality, and cost-effectiveness will improve outcomes of AMS programs across the region.

Acknowledgments. L.S.P.M. acknowledges academic support from the National Institute of Health Research (NIHR) Imperial Biomedical Research Centre (BRC) and the National Institute for Health Research Health Protection Research Unit (HPRU) in Healthcare Associated Infection and Antimicrobial Resistance at Imperial College London in partnership with Public Health England. Weber Shandwick Hong Kong is gratefully acknowledged for providing editorial support.

Financial support. Y.D. acknowledges public research funding from the National Institutes of Health, Japan Agency for Medical Research and Development. Editorial support for Weber Shandwick Hong Kong was funded by bioMérieux.

Conflicts of interest. L.S.P.M. has consulted for and/or received speakers fees from bioMérieux (2013-2020), DNAelectronics (2015), Dairy Crest (2017-2018), Eumedica (2016-2020), Pfizer (2018-2020), and Umovis Labs (2020) and has received research grants from the National Institute for Health Research (2013-2019), Leo Pharma (2016), and CW + Charity (2018-2020. Y.D. has consulted for Gilead, Shionogi, Janssen, Entasis, VenatoRx, bioMerieux, and received speaking fees from MSD, AstraZeneca, FujiFilm Toyoma Chemical, Pfizer, MSD, Janssen, Shionogi, Astellas and Kanto Chemical. A.L.H.K. has received fees from bioMérieux, Pfizer, Menarini and ThermoFisher Scientific. Q.C. has received fees from bioMérieux. All other authors report no conflicts of interest relevant to this article.

\section{References}

1. Beganovic M, McCreary EK, Mahoney MV, et al. Interplay between rapid diagnostic tests and antimicrobial stewardship programs among patients with bloodstream and other severe infections. J Appl Lab Med 2019;3: 601-616.

2. Barlam TF, Cosgrove SE, Abbo LM, et al. Executive summary: implementing an antibiotic stewardship program: guidelines by the Infectious Diseases Society of America and the Society for Healthcare Epidemiology of America. Clin Infect Dis 2016;62:1197-1202.

3. Antimicrobial stewardship programmes in healthcare facilities in low- and middle-income countries. A WHO practical tool kit. World Health Organization website. https://apps.who.int/iris/bitstream/handle/10665/ 329404/9789241515481-eng.pdf?sequence=1\&isAllowed=y. Published 2019. Accessed March 9, 2021.

4. Fong K. The impact of rapid diagnostic testing and biomarkers on antimicrobial stewardship: deriving their optimal benefit. Infectious Disease Special Edition website. www.idse.net/Review-Articles/Article/10-20/The-Impactof-Rapid-Diagnostic-Testing-and-Biomarkers-on-Antimicrobial-Steward ship-Deriving-Their-Optimal-Benefit/60848. Published November 2020. Accessed March 9, 2021.

5. Timbrook TT, Morton JB, McConeghy KW, et al. The effect of molecular rapid diagnostic testing on clinical outcomes in bloodstream infections: a systematic review and meta-analysis. Clin Infect Dis 2017;64: $15-23$.

6. Timbrook TT, Spivak ES, Hanson KE. Current and future opportunities for rapid diagnostics in antimicrobial stewardship. Med Clin North Am 2018;102:899-911.

7. Park SY, Chang HH, Kim B, et al. Human resources required for antimicrobial stewardship activities for hospitalized patients in Korea. Infect Control Hosp Epidemiol 2020;41:1429-1435.

8. Patel TS, Kaakeh R, Nagel JL, Newton DW, Stevenson JG. Cost analysis of implementing matrix-assisted laser desorption ionization-time of flight mass spectrometry plus real-time antimicrobial stewardship intervention for bloodstream infections. J Clin Microbiol 2016;55: 60-67.

9. Pliakos EE, Andreatos N, Shehadeh F, Ziakas PD, Mylonakis E. The costeffectiveness of rapid diagnostic testing for the diagnosis of bloodstream infections with or without antimicrobial stewardship. Clin Microbiol Rev 2018;31:e0095-17.

10. Kim B, Lee MJ, Moon SM, et al. Current status of antimicrobial stewardship programmes in Korean hospitals: results of a 2018 nationwide survey. J Hosp Infect 2020;104:172-180.

11. Na SH, Kim CJ, Kim M, et al. Impact of the multiplex polymerase chain reaction in culture-positive samples on appropriate antibiotic use in patients with staphylococcal bacteremia. Diagn Microbiol Infect Dis 2016;84: 353-357.

12. Suzuki H, Hitomi S, Yaguchi Y, et al. Prospective intervention study with a microarray-based, multiplexed, automated molecular diagnosis instrument (Verigene system) for the rapid diagnosis of bloodstream infections, and its impact on the clinical outcomes. J Infect Chemother 2015;21: 849-856.

13. Niwa T, Yonetamari J, Hayama N, et al. Clinical impact of matrixassisted laser desorption ionization-time of flight mass spectrometry combined with antimicrobial stewardship interventions in patients with bloodstream infections in a Japanese tertiary hospital. Int J Clin Pract 2019;73:e13332.

14. Nomura F, Tsuchida S, Murata S, Satoh M, Matsushita K. Mass spectrometry-based microbiological testing for blood stream infection. Clin Proteomics 2020;17:14.

15. Tarai B, Das P. FilmArray meningitis/encephalitis (ME) panel, a rapid molecular platform for diagnosis of CNS infections in a tertiary-care hospital in North India: One-and-half-year review. Neurol Sci 2019;40: 81-88.

16. Nadjm B, Dat VQ, Campbell JI, et al. A randomised controlled trial of matrix-assisted laser desorption ionization-time of flight mass spectrometry (MALDITOF-MS) versus conventional microbiological methods for 
identifying pathogens: Impact on optimal antimicrobial therapy of invasive bacterial and fungal infections in Vietnam. $J$ Infect 2019;78: 454-460.

17. Pulcini C, Morel CM, Tacconelli E, et al. Human resources estimates and funding for antibiotic stewardship teams are urgently needed. Clin Microbiol Infect 2017;23:785-787.

18. Morris AM, Rennert-May E, Dalton B, et al. Rationale and development of a business case for antimicrobial stewardship programs in acute care hospital settings. Antimicrob Resist Infect Control 2018;7:104.
19. Cox JA, Vlieghe E, Mendelson M, et al. Antibiotic stewardship in low- and middle-income countries: the same but different? Clin Microbiol Infect 2017;23:812-818.

20. Rawson TM, Moore LSP, Zhu N, et al. Bacterial and fungal coinfection in individuals with coronavirus: a rapid review to support COVID-19 antimicrobial prescribing. Clin Infect Dis 2020;71:2459-2468.

21. Shin DH, Kang M, Song KH, et al. A call for antimicrobial stewardship in patients with COVID-19: a nationwide cohort study in Korea. Clin Microbiol Infect 2020. doi: 10.1016/j.cmi.2020.10.024. 